Cinémas

Revue d'études cinématographiques

Journal of Film Studies

\title{
Les répétitions contrariées. Sur Beau travail de Claire Denis Thwarted Regularities
}

\section{Caroline Renard}

Volume 23, numéro 1, automne 2012

URI : https://id.erudit.org/iderudit/1013368ar

DOI : https://doi.org/10.7202/1013368ar

Aller au sommaire du numéro

\section{Éditeur(s)}

Cinémas

ISSN

1181-6945 (imprimé)

1705-6500 (numérique)

Découvrir la revue

Citer cet article

Renard, C. (2012). Les répétitions contrariées. Sur Beau travail de Claire Denis. Cinémas, 23(1), 55-71. https://doi.org/10.7202/1013368ar

\section{Résumé de l'article}

Beau travail de Claire Denis fait partie des films qui nous invitent à les aborder sous l'angle de la répétition. Figure esthétique majeure des arts $\mathrm{du} \mathrm{xx}^{\mathrm{e}}$ siècle, la répétition peut aussi être une forme discrète qui circule d'image en image. Parfois elle se cache et se déguise d'un plan à l'autre au sein d'un même film. Dans une approche analytique, l'auteure de cet article emploie la notion de "plan de consistance " présentée par Gilles Deleuze et Félix Guattari dans Mille plateaux (1980) pour proposer une étude figurative des formes de la répétition dans Beau travail. Deleuze et Guattari ont défini le "plan de consistance » comme un espace formel qui est simultanément un fond et un volume, une armature et une trame. Cet espace résiste dans la durée. En termes de cinéma, le plan de consistance d'un film est élaboré par l'articulation des plans, par la composition des cadres, par les mouvements qui animent l'espace ou les trajets qui le parcourent. Il constitue un repère visuel parfois fuyant, en mouvement mais repérable. L’analyse de Beau travail montre que les répétitions, loin de simplement construire des effets narratifs ou scéniques, participent de la mise en place d'un socle perceptif à la fois récurrent et variable, d'une trame visuelle reconnaissable et instable. L'auteure met ainsi au jour, dans l'économie figurative de ce film d'inspiration littéraire et musicale, l'élaboration de ce " plan de consistance » comme fond visible et invisible du montage filmique.
Ce document est protégé par la loi sur le droit d'auteur. L'utilisation des services d'Érudit (y compris la reproduction) est assujettie à sa politique d'utilisation que vous pouvez consulter en ligne.

https://apropos.erudit.org/fr/usagers/politique-dutilisation/ 


\title{
Les répétitions contrariées. Sur Beau travail de Claire Denis
}

\section{Caroline Renard}

\begin{abstract}
RÉSUMÉ
Beau travail de Claire Denis fait partie des films qui nous invitent à les aborder sous l'angle de la répétition. Figure esthétique majeure des arts du $\mathrm{XX}^{\mathrm{e}}$ siècle, la répétition peut aussi être une forme discrète qui circule d'image en image. Parfois elle se cache et se déguise d'un plan à l'autre au sein d'un même film. Dans une approche analytique, l'auteure de cet article emploie la notion de "plan de consistance" présentée par Gilles Deleuze et Félix Guattari dans Mille plateaux (1980) pour proposer une étude figurative des formes de la répétition dans Beau travail. Deleuze et Guattari ont défini le "plan de consistance» comme un espace formel qui est simultanément un fond et un volume, une armature et une trame. Cet espace résiste dans la durée. En termes de cinéma, le plan de consistance d'un film est élaboré par l'articulation des plans, par la composition des cadres, par les mouvements qui animent l'espace ou les trajets qui le parcourent. Il constitue un repère visuel parfois fuyant, en mouvement mais repérable. L'analyse de Beau travail montre que les répétitions, loin de simplement construire des effets narratifs ou scéniques, participent de la mise en place d'un socle perceptif à la fois récurrent et variable, d'une trame visuelle reconnaissable et instable. L'auteure met ainsi au jour, dans l'économie figurative de ce film d'inspiration littéraire et musicale, l'élaboration de ce "plan de consistance» comme fond visible et invisible du montage filmique.
\end{abstract}

Claire Denis, dans Beau travail (2000), aurait très bien pu faire de la répétition une figure pure et mécanique. En effet, la description d'un rapport de force entre trois hommes au sein d'un régiment de la légion étrangère appellerait aisément des répétitions stéréotypées. Or, repérer et décrire ${ }^{1}$ les répétitions dans ce film nous invite à constater qu' elles construisent des figures complexes qui interviennent moins en termes de fonction qu'en termes d'affection: elles affectent littéralement le film, les personnages et les 
spectateurs. Tout en possédant des fonctions narratives d'ancrage du récit ou des fonctions scéniques de repérage spatial, la répétition touche majoritairement les personnages dans leur corps et participe de leurs actions ${ }^{2}$. Elle a par ailleurs sur les spectateurs un effet directement sensible. Mais nous nous intéresserons surtout à la manière dont elle affecte le champ de forces et d'intensités que constitue le film. Dans le tissage de matériaux visuels et sonores qu'il compose, la répétition joue un rôle structurel. Il ne s'agira donc pas pour nous d'analyser le rôle narratif de la répétition, dont nous ne négligeons pas l'importance; il ne s'agira pas non plus de penser sa fonction scénique dans la construction d'une géographie imaginaire. Nous nous éloignerons également de l'analyse de la fonction psychique du répété et de ses prolongements en termes psychanalytiques. Si chacune des fonctions de la répétition possède des enjeux et des effets distincts et spécifiques dans le film et pour les spectateurs, nous nous attacherons seulement à analyser la manière dont les reprises mises en place par Claire Denis construisent ce que Gilles Deleuze et Félix Guattari (1980) pourraient nommer un "plan de consistance», c'est-à-dire un espace formel, une structure qui est à la fois une trame, un fond et un volume. Le plan de consistance d'un film forme un champ visuel permanent sur lequel la pensée du spectateur projette les actions, les dialogues et les rapports entre les personnages. Il est élaboré morceau par morceau par la composition des cadres, par les mouvements visuels qui animent l'espace ou les trajets qui le parcourent. C'est un repère plastique constant et pourtant instable, une forme mouvante mais reconnaissable, un fond résistant. Les répétitions discrètes construisent dans Beau travail un fond stratifié: aussi présent mais aussi léger qu'une pièce de tissu agitée par un souffle de vent ${ }^{3}$.

Le roman d'Herman Melville, Billy Budd, marin (1987 [1924]), sert de terreau à la trame narrative du film. Le récit dans lequel l'auteur américain décrit l'affection ascétique d'un capitaine de navire pour un jeune mousse est transposé dans l'univers de la Légion étrangère stationnée à Djibouti. Le personnage de Galoup, interprété par Denis Lavant, est le narrateur. Il est aussi le fil conducteur du film. Il repense aux raisons pour lesquelles il a été démis de ses fonctions d'adjudant-chef. 
Son récit d'introspection se termine par le suicide probable du personnage. Allongé sur le lit qu'il vient de refaire au carré, il pose son revolver à plat sur son thorax. Dans son monologue intérieur, il prononce alors les mots qu'il porte tatoués sur la poitrine: "Sers la bonne cause et meurs.» Sur son bras replié, cadré en gros plan, le sang qui circule dans ses veines devient le seul mouvement qui anime l'image. Dans le plan large qui suit, sur la piste d'un night-club, Galoup entame une danse hors du temps, solitaire, physique et déchaînée sur la chanson The Rhythm of the Night (Corona). Du micromouvement vasculaire à l'ampleur du geste de la danse, le mouvement est au cœur des préoccupations figuratives de ce film.

"Je ne regarde qu'aux mouvements", pourrait dire Claire Denis en reprenant à son compte les mots de Søren Kierkegaard (1984, p. 52). Ne regarder qu'aux mouvements. Ici les mouvements de corps, les déplacements des légionnaires (un train, un groupe d'hommes qui marchent) forment une animation visuelle, mais la cinéaste décrit aussi les mouvements des affects, la variation des états intérieurs ainsi que le trajet des sensations. C'est l'essence du cinéma que d'enregistrer et de restituer le mouvement d'un objet visuel. Claire Denis revisite cette vertu filmique. Tout en travaillant sur le geste ou sur la chorégraphie des ensembles filmés ${ }^{4}$, elle soulève la question de ce qu'est un mouvement en soi au cinéma, un mouvement en dehors des corps, indépendant des actions, hors des trajets. Elle semble rejoindre ici le projet du cinéma rythmique de certaines avant-gardes des années 1920 dont on connaît les liens à la danse ${ }^{5}$. En 1928, le critique Roland Guerard insistait déjà sur l'importance du mouvement dans les films, qui justifiait pour lui que l'on associe plutôt le cinéma aux arts de la danse qu'à ceux de la musique. C'est ce lien du cinéma à la rythmique gestuelle que semble renouer Claire Denis dans Beau travail, rappelant que le cinéma est pour certains avant tout "affaire de mouvement» (Guerard 1928).

Gilles Deleuze et Félix Guattari (1980, p. 344) dans Mille plateaux notaient que:

Le mouvement est dans un rapport essentiel avec l'imperceptible, il est par nature imperceptible. C'est que la perception ne 
peut saisir le mouvement que comme la translation d'un mobile ou le développement d'une forme.

Pour atteindre directement au mouvement, pour se débarrasser du médiateur qu'est l'objet mobile, Claire Denis utilise la répétition comme instrument d'observation privilégié. Elle observe et elle met au jour ce qui dans des mouvements différents se répète. Elle filme la répétition d'une même forme à travers des mobiles multiples pour donner à voir le mouvement lui-même.

Le rythme de vie des légionnaires, leurs activités d'entraînement, leurs gestes quotidiens construisent des actions répétitives montrées à plusieurs reprises ${ }^{6}$. Si nous considérons comme répétition pure le retour à l'identique de la chose elle-même, une répétition "nue» (Deleuze 1968), transparente, sans parasite qui menacerait sa totalité, Beau travail n'en contient aucune. Les légionnaires font toujours la même chose, mais ils le font toujours différemment et jamais au même rythme. Loin d'un surplace répétitif, le film affirme au contraire un trajet. Bien que construit en accolade entre deux scènes qui se passent dans un dancing, il raconte des parcours. Celui, intérieur, que vivent les personnages, mais aussi celui, spatial, qui les fait évoluer de l'onde marine à l'aridité d'un lac de sel $^{7}$.

Le film possède un aspect assez peu répétitif. Il glisse d'une action à l'autre dans une structure narrative qui entremêle les souvenirs de Galoup à Djibouti et son retour à la vie civile à Marseille. Mais ce montage parallèle tend moins à jouer sur des temporalités différentes qu'à affirmer l'unité de conscience de ce personnage rigide dont la jalousie va être mise à l'épreuve. Lors d'un entraînement de son bataillon en mer, un accident d'hélicoptère a lieu. Le jeune Sentain, nouvelle recrue, sauve un de ses camarades de la noyade. La reconnaissance de la bravoure de Sentain par le commandant Forestier déclenche la jalousie de l'adjudant Galoup. Par ruse, et voulant faire croire à une désertion du jeune homme, il envoie Sentain dans le désert avec une boussole sournoisement truquée. La réalité de son intention sera mise au jour et lui vaudra d'être exclu de la Légion ${ }^{8}$.

Claire Denis travaille par bribes. Parfois, un seul plan vaut pour une scène; une phrase, pour tout un dialogue. Elle reste 
ainsi fidèle à la frugalité narrative de Melville. Elle monte son récit par brefs fragments. Elle ne construit pas un ensemble, mais accumule les litotes. Elle ne fabrique pas non plus une totalité. Ses cadres sont serrés, les plans rapprochés, les corps filmés par morceaux; le paysage est un détail avant d'être une étendue. La ville, elle, est filmée comme recoins ou comme intérieurs, jamais comme un territoire. Des lieux, le film extrait les couleurs, les lignes, les textures. De Djibouti, Claire Denis élimine tout ce qui pourrait permettre de reconnaître la ville, sauf son nom répété dans un plan bref par un policier qui crie au téléphone: "Ici Djibouti. Allô, Djibouti.» Le nom est une géographie à lui seul. Il en sera de même pour Marseille. Nommée une seule fois, la ville est l'autre espace du film, son contrechamp. De la même manière, Claire Denis résume la vie nocturne de ses légionnaires à un dancing. Des visages de femmes et de soldats qui dansent devant un mur couvert de miroirs. Rien d'autre n'est décrit. Elle réduit également la légion à quelques hommes, un vieux char rouillé, des vêtements vert kaki sur une corde à linge. Elle élimine toutes les choses qu'elle n'a pas. Économiquement, le film ne pouvait réunir de légion entière, ni de chars d'assaut, d'armes ou d'avions de combat. Pourtant, elle met tout: Djibouti, Marseille, la Légion. Tout loge dans les corps, dans le maintien des épaules, dans un platane ou une tente près de la mer. Tout est là dans des cadres serrés, sursaturés de textures visuelles.

Claire Denis maintient la tension narrative dans l'absence de développement des séquences. Ainsi, par exemple, celle qui décrit les causes de la punition de Sentain est-elle filmée en cinq plans. 1) Sentain tend une gourde à un autre légionnaire qui creuse une fosse en plein soleil. 2) L'adjudant Galoup donne un coup de pied dans la gourde. Sentain a juste le temps de dire à son supérieur qu'il le trouve injuste et Galoup le gifle. 3) Sentain lève alors le poing gauche et frappe Galoup qui s'écroule. 4) Le commandant Forestier observe ces événements de loin. 5) Sentain est emmené hors du campement par Galoup '. Traiter ainsi une séquence en cinq plans permet de glisser sur les actions sans aucune insistance, tout en jouant d'une esthétique fragmentaire et d'un rythme sec et rapide. Sans que cela nuise à l'homogénéité du récit. 
Beau travail s'articule autour d'une série de mouvements récurrents, repris et modifiés progressivement d'une scène à l'autre. C'est à travers eux que s'élabore lentement ce que nous proposons de considérer comme le plan de consistance du film. Sans prétendre à l'exhaustivité, nous travaillerons sur trois de ces mouvements. Le premier est un geste latéral : trajet de ce qui passe dans le cadre sans y rester. Le deuxième questionne la profondeur de champ: c'est celui qui anime les perspectives visuelles. Le troisième, quant à lui, est concentrique: mouvement de tension dramatique ancré au milieu du cadre.

Le premier de ces mouvements est impulsé au film dès le premier plan qui apparaît pendant le générique. Un travelling latéral sur un mur décrit une fresque. Elle représente en noir sur rouge la silhouette d'un groupe de soldats sur une colline. À cette scène de guerre figée répond en son off un chœur de légionnaires. Le mouvement latéral de la caméra annonce un des principes du film: les choses vont entrer dans le cadre sans y rester. Ce mouvement est à lui seul une question de cinéma: comment sur un fond, filmé plein cadre, faire venir des figures? Beau travail est un film dont la surface visuelle repose sur un fond, comme en peinture un motif est posé sur un arrière-plan. L'enjeu est de faire venir des corps, des objets, des visages, des couleurs sur ce fond. Apparaîtront ainsi dans le cadre: le dancing, Djibouti, un paysage vu du train, des corps en exercice, des visages de femmes, des visages de légionnaires. Tous passent dans le champ sans que le film leur donne véritablement le temps de l'habiter, d'y inscrire une présence diégétique forte. Comme fond, au mur de ce premier plan se substitueront plus tard la mer, la terre sèche et rocailleuse de Djibouti, un lac de sel.

La traversée latérale du cadre revient de différentes manières. Dans un travelling filmé depuis la mer sur la berge où les soldats ont installé leur étendoir, tout est fait pour souligner l'horizontalité de la composition. Une corde à linge, la terre et le bord de l'eau forment des lignes parallèles qui traversent le cadre. Plus loin, ce sont des cordes qui tracent des horizontales dans l'image. Les soldats doivent traverser l'espace en se faisant glisser sur trois cordes, au plan suivant, ils doivent marcher sur un filin en se tenant par les mains à une autre corde au-dessus de leur 
tête. Dans cette position de figurines découpées, ils traversent le cadre. Avec un certain humour, Claire Denis filme presque les soldats comme du linge qui sèche. Au plan suivant, un travelling sur une lessive étendue prolonge cette impression. Quelques plans plus loin, un portique formé de deux barres horizontales est placé au premier plan tandis que les légionnaires repassent devant leur caserne. La cinéaste inscrit ainsi une forme qui problématise trois éléments cinématographiques: la composition du cadre, l'entrée des figures dans le champ et l'analyse du mouvement par le regard. Dans la deuxième moitié du film, ce mouvement latéral est repris. Ainsi les légionnaires traversent-ils un plateau volcanique de gauche à droite avec chacun une pelle ou une pioche sur l'épaule. Un peu plus tard, c'est la troupe menée par Galoup qui redessine cette ligne sur un fond rocailleux, en marchant au pas cadencé sur un chemin de terre. Bien sûr, le tracé de la ligne varie. Au lieu de suivre le chemin, Galoup entraîne les hommes sur un talus. La caméra est parfois en position latérale, parfois légèrement de biais. Penser le mouvement et envisager l'entrée d'un motif dans le champ filmique font partie des enjeux figuratifs du cinéma. Claire Denis développe ce geste latéral, elle en fait une ligne suspendue, un tracé légèrement oblique ou un mouvement sinusoïdal.

Le deuxième mouvement qu'explore Beau travail est aussi un geste classique de cinéma, c'est le mouvement de ce qui vient de la profondeur de champ. Un objet avance vers l'avant-plan dans un cadre vide. Claire Denis revisite la ligne de fuite tracée par de nombreux mobiles de l'histoire du cinéma et elle la rend plus malléable. Dans une scène d'entraînement militaire, les soldats effectuent un parcours qui met leur force et leur souplesse à l'épreuve. Une série d'exercices les conduit à ramper sous des barbelés, passer au-dessus d'une barre fixe, faire une course d'obstacles, franchir un mur, sauter dans une fosse et en ressortir... Certains parcours sont filmés en plongée, de biais ou de profil, mais l'un d'eux est filmé de face, dans la profondeur de champ. Il s'agit pour les soldats d'effectuer une course de haies au cours de laquelle ils doivent sauter par-dessus une poutre et se faufiler en dessous d'une barre placée plus bas. Les légionnaires traversent successivement le champ vers l'avant. Le point 
de vue, placé dans un premier temps à hauteur de regard, se déplace peu à peu de haut en bas au gré de légers panoramiques verticaux qui accompagnent le saut et l'accroupissement successifs des soldats. Lors du passage du dernier, la caméra est presque au ras du sol. Les corps sont filmés comme des vagues, ils s'enroulent autour des obstacles comme dans un vaste mouvement de ressac. Or, ce geste que chacun répète après l'autre et qui contamine la caméra devient assez vite un geste anonyme et un mouvement pur. Comme l'écrivent Gilles Deleuze et Félix Guattari (1980, p. 308) :

Chacun s'avance comme une vague, mais sur le plan de consistance, c'est une seule et même Vague abstraite dont la vibration se propage suivant la ligne de fuite ou de déterritorialisation qui parcourt tout le plan. [...] Les vagues sont les vibrations, les bordures mouvantes qui s'inscrivent comme autant d'abstractions sur le plan de consistance.

Chaque soldat effectue le même mouvement que les autres: course de bosses, passage par-dessus des barres, saut dans une fosse et franchissement d'un mur. Chaque corps a son mouvement propre, mais chacun est traversé par un trajet commun, une énergie partagée, un geste collectif. Le plan de consistance est particulièrement apparent dans les plans où ce mouvement traverse la profondeur de champ. On le voit, corps après corps, s'y inscrire, se répéter et se propager sur une ligne de regard qui traverse le film pour atteindre directement son spectateur.

Le troisième élément qui nous intéresse engage moins une traversée ou un déplacement qu'un mouvement dans le cadre, sur l'espace scénique lui-même; il peut dépendre de la scénographie, mais parfois d'un geste. Limité par les bords du cadre, il est peutêtre le plus théâtral. Ce mouvement est circulaire, dramatiquement concentrique même. Il s'ancre au milieu de l'image pour y désigner une exigence, une tension, un rapport de force. C'est par exemple le mouvement de la troupe de légionnaires regroupés au centre de l'image. Ils obéissent à l'ordre de l'adjudant Galoup de creuser et d'aplanir un terrain rocailleux au milieu de nulle part pour y réparer une route effacée. Au commandement de leur chef, les hommes commencent à abattre leurs outils. 
S'élève alors un nuage de poussière blanche sur l'horizon maritime. Un peu plus tard dans le même paysage, les hommes s'exercent à nouveau. Les légionnaires effectuent une sorte de danse guerrière autour de Galoup. Les hommes piétinent sur place en formant un cercle. Galoup tourne sur lui-même au centre du cercle, s'avance brutalement vers l'un ou vers l'autre avant de faire un pas de recul pour reprendre sa place. Le mouvement est à la fois étoilé et circulaire.

C'est dans un face à face silencieux que ce mouvement est le plus développé. Après un exercice de corps à corps où, deux par deux, les hommes se propulsent, thorax contre thorax, dans une étreinte guerrière (autre mouvement latéral que cette rencontre de forces contraires), Galoup et Sentain amorcent un duel chorégraphique. Il s'agit d'un exercice d'entraînement au combat, mais cette fois, le jeu dépasse le cadre militaire. La scène est tout d'abord filmée en plan très large. Les deux hommes se regardent et amorcent un large cercle autour d'un axe imaginaire; peu à peu, ils se rapprochent, jusqu'à être face à face, presque immobiles, à quelques centimètres l'un de l'autre. Le montage les enferme dans un découpage qui se resserre sur eux sans que la tension explose. En off, les voix du chœur de l'opéra Billy Budd de Benjamin Britten, composé sur le motif narratif de l'œuvre de Melville, soulignent la tension qui anime les deux hommes; elles accompagnent le trajet circulaire de la position de la caméra. Ce fond visuel qui enserre le centre de l'image est également présent dans la scène qui conduit Sentain, poussé à bout, à frapper son adjudant-chef. Un des légionnaires est cadré, épuisé, les mains en sang, au fond de la fosse qu'il a été condamné à creuser en plein soleil. Ces plans sont ceux qui exacerbent la tension entre Galoup et Sentain. La circularité qui se creuse et les affects qui se tendent sont comme des images dans la pensée du spectateur. Le piège qu'avait imaginé Galoup s'est refermé sur Sentain. Un gros plan sur la boussole sabotée par celui-là répétera d'une manière encore détournée ce même fond visuel. Cadrée en gros plan, la boussole montre une aiguille qui s'affole en toutes directions, comme lorsque Galoup, au centre du cercle, avançait d'un pas saccadé vers l'un ou l'autre de ses hommes choisis de manière aléatoire. 
Si le premier mouvement que nous venons de décrire peut figurer un geste d'appropriation de l'espace (c'est par lui que les légionnaires affirment leur présence sur le territoire de Djibouti, comme c'est par lui qu'ils deviennent des figures cinématographiques), le deuxième creuse plutôt dans l'univers filmique un trajet, un lieu d'existence des corps mobiles. Le troisième, quant à lui, désigne une forme qui figure des rapports de force. Trois mouvements, des reprises. Chacune de ces reprises engage des objets différents. Cachées, ces répétitions discrètes ont pourtant quelque chose d'hypnotique, qui retient le regard puis qui l'habite. S'agit-il de simples échos figuratifs ou d'une sorte de refrain visuel repris en canon, comme le sont certains motifs de l'opéra de Benjamin Britten? Ces mouvements sont loin de former le tout du film; ils en dessinent des lignes de force. Ces reprises plastiques indiquent au spectateur que le sujet de Claire Denis est autant la vie des légionnaires que la plastique, la malléabilité et le mouvement qui animent la surface des images.

Deux plans montés à la suite l'un de l'autre décrivent successivement la surface de la mer et une page de journal intime, ressac et écriture. Par surimpression, l'écriture se pose sur le scintillement de la lumière à la surface de l'eau. Ce mélange d'images offre une configuration particulière de la surface filmique. Mais cette stratification, au lieu de sédimenter des textures, au lieu d'accumuler de la matière, souligne l'irréductibilité des strates entre elles. La clarté de la lumière ne peut se confondre avec l'encre noire, et le mouvement impermanent des reflets s'oppose à la marque indélébile de l'écriture. Une strate ne répète ni ne consolide la suivante. Il y a du jeu entre chaque surface, des articulations entre les strates, des successions d'épaisseurs ou de textures. C'est la ramification de ces strates, la superposition des répétitions plastiques précédemment décrites, le plissement constant des différents mouvements les uns sur les autres, qui compose le plan de consistance du film. C'est en lui que s'agence la diversité des mouvements.

Les régularités visuelles ainsi mises en place sont régulièrement contrariées par d'autres formes qui viennent les annuler ou les contredire. Ces répétitions, à la fois quotidiennes et visuelles, s'opposent tout d'abord à une force qui est de l'ordre du hasard. 
Préparer les corps, les exercer, les entraîner, reproduire les mêmes gestes au même rythme ne stabilise ni la position ni le devenir des personnages. La répétition mécanique rencontre le hasard et ce dernier la contredit. Ce blocage vient du second personnage central, Sentain, qui est celui dont le parcours est le plus énigmatique. Sentain arrive dans ce corps de légion sans motivation apparente. Il est un enfant trouvé ${ }^{10}$. Lorsqu'il se perd à cause de la malveillance de Galoup, c'est le hasard qui sauve deux fois le jeune homme. Une première fois, le hasard intervient lorsqu'un de ses camarades reconnaît sa boussole rongée par le sel sur l'étalage d'un marchand. En effet, cette découverte permet de dénoncer l'acte de sabotage de Galoup et de le faire condamner. C'est également par hasard que Sentain est trouvé par une caravane et qu'il est ramené vers la ville. Sentain est donc le personnage que l'on trouve. Il est la trouvaille, celui sur qui on tombe par hasard et par chance et qui rend heureux celui qui l'a découvert (la "belle trouvaille»); c'est celui sur qui la répétition a le moins de prise.

Son trajet s'inscrit pourtant sur les fonds visuels que nous avons décrits. Il parcourt les mêmes lignes que les autres. Il entre dans l'espace des tensions, mais il en modifie parfois le rythme ou il en inverse le sens. Ainsi, lorsqu'il est puni pour avoir frappé son supérieur et emmené dans le désert d'où il doit revenir seul, Sentain n'est plus qu'un point dans un immense paysage. Il s'avance vers la caméra. Il est si loin qu'il se distingue à peine parmi les rochers. Des bosquets dessinent une diagonale que plus aucun mouvement n'anime. Au plan suivant, grâce à un raccord dans l'axe, une ligne de chemin de fer s'inscrit sur le même tracé. Cette ligne répond au regard du spectateur qui croit retrouver l'axe du plan précédent. Or le train, au lieu de s'avancer vers nous, dans la même direction que Sentain, s'éloigne dans la profondeur de champ et disparaît en transformant le raccord dans l'axe en faux raccord de mouvement. Si la ligne de fuite est maintenue, l'orientation du mobile est inversée, signifiant ainsi la perte de Sentain. Plus loin, ce sera finalement du fond du champ qu'arrivera, après un large virage, la caravane des marchands de sel qui retrouvera et sauvera Sentain. Comme si le trajet que parcourt cette diagonale retrouvait une 
direction originelle. Bien que le mobile ait changé (les chameliers et leurs montures au lieu des légionnaires), bien que le rythme soit différent et que le territoire ne soit plus le même, le regard reconnait dans cette trajectoire un des socles visuels du film. Claire Denis ne se contente donc pas de poser des lignes de mouvement ni de construire un fond visuel. Travailler une forme à son terme, c'est aussi l'effacer, la contredire, provoquer l'effondrement du plan de consistance et le reconstruire.

Le film dessine également une autre ligne d'opposition diégétique et rythmique à ces régularités. Aux travaux répétitifs des soldats, Claire Denis oppose l'immobilité contemplative des populations civiles de Djibouti. Les activités d'entraînement de la Légion sont souvent observées avec curiosité, mais elles s'opposent surtout au rythme modéré de la population souligné par des plans de visages. La majorité de ces plans décrit des visages de femmes: celles qui dansent avec les légionnaires, mais aussi des passantes. Quatre visages de femmes qui portent des voiles colorés et qui rient à l'angle d'une rue. Le visage d'une marchande de khat apparaît en gros plan. Des visages de femmes traversent le paysage. Une bergère garde son troupeau près de la voie ferrée. La passagère d'un train subit impassiblement son voyage. Deux plans sont particulièrement frappants. Ce sont deux plans au ralenti sur le visage d'une jeune femme qui danse dans la lumière rouge du night-club. Dans les deux cas, ces plans sont raccordés au personnage de Galoup. Le visage est celui de la femme qu'il fréquente. La première scène se passe une nuit de sortie: "C'était le début du ramadan, dit Galoup en voix off. Toutes les arcades étaient illuminées. " Le commandant Forestier circule en ville dans une voiture, il croise ses hommes, puis Galoup, seul. Un travelling avant depuis la voiture avance sur la rue et s'arrête en plan moyen sur Galoup probablement ivre; en contrechamp, un plan très bref de Forestier décrit la surprise du commandant, puis un plan nettement plus long s'attarde sur le visage de cette femme qui danse face à la caméra dans l'éclairage rouge clignotant du dancing. Une musique très lente, sourde, un peu électrique, densifie l'étrangeté de ce ralenti. Plus loin, après une longue séquence sur la corvée de linge des soldats, tandis que Galoup repasse, la même sonorité fait retour. Elle est 
à nouveau associée au visage de la même femme cadrée cette fois en gros plan dans la lumière rouge, regard fixé sur la caméra, dans un ralenti qui évoque une opacification de la perception.

Il est clair que ce visage revient comme une obsession de la pensée de Galoup, toujours accompagné de cette musique lointaine et mystérieuse, dans un moment d'égarement du personnage ou de retour sur soi. Non seulement ces répétitions sonores et visuelles totalement détachées du reste de l'économie figurative du film introduisent un rythme différent, mais elles reflètent aussi la position incertaine du personnage central. Toute l'attention accordée à la routine dans les activités quotidiennes cache la fragilité, l'incertitude identitaire, l'insatisfaction et la faiblesse de Galoup. Lorsque, épuisé par une marche avec ses hommes, il s'endort à une table, face à la mer, la même sonorité étrange s'étire une troisième fois sur une image au ralenti. Il s'agit d'un travelling sur un troupeau de chèvres au bord de la route. Le regard caméra de la femme qui les surveille renvoie immédiatement à celui de la danseuse de Djibouti, mais il s'agit ici d'un regard de mépris que renforce le geste de cette femme inconnue: elle jette des cailloux en direction de la caméra. Plan subjectif, image-souvenir ou image-rêve, ce plan déstabilise le point de vue du film. Ces trois plans au ralenti créent d'étranges ruptures de rythme.

Le graphisme d'un plan du début du film rompt lui aussi avec le reste des images. À Marseille, Galoup escalade un énorme platane. Il en élague les jeunes pousses à coups de machette comme on taillerait des roseaux. Le tronc puissant de l'arbre et la masse gris clair et solide des branches principales entrent en contraste avec les légères lignes noires des petits branchages à supprimer. Ce plan au mouvement complexe ne correspond à aucune autre configuration visuelle. C'est à ce moment-là qu'en voix off, Galoup répète cette phrase ambiguë: "Peut-être qu'avec les remords commence la liberté. Peut-être qu'avec les remords commence la liberté.» De quelle liberté parle-t-il en ce début de film? Qu'est-ce que la liberté pour un ex-légionnaire? Et qu'est-ce que les remords libèrent de la pensée? S'agit-il juste de l'ambition d'un adjudant-chef? Ou alors s'agit-il d'une liberté de geste, celle qui, justement, permet à Galoup de se détacher 
de toutes les régularités mécaniques de son image de militaire, pour entrer dans ce mouvement extraordinaire décrit par la danse des derniers plans du film? Lui, dont le corps est le plus strict, le plus rigoureux du film, sans cesse étiré et tendu, faisant honneur aux plis de son uniforme, invente soudain un geste démesuré, un laisser-aller total du corps. Il écoute d'abord la musique, il en suit un peu le rythme, puis, comme une explosion, il décharge toute la tension qui est en lui. L'espace se remplit alors du mouvement qui traverse ses membres. Car ce n'est plus lui qui semble agir mais l'ensemble des parcours qu'il a effectués jusque-là. Le mouvement déborde de toutes parts. Les mouvements concentrés par les différentes stratifications visuelles du film excèdent désormais les limites de son corps. Il n'y a plus ni forme, ni organisation, ni expression, juste un mouvement pur qui anime et traverse l'espace dans cette désarticulation totale et cet effondrement des rythmes réguliers.

La répétition dans Beau travail n'est ni une action ni un détail, à peine une figure, juste une trame légère. Le récit joue de détours, de décalages, de caches qui, loin de suspendre la répétition, l'entourent, la protègent, la réinventent sans cesse. Tout un système plastique et formel complexe permet ce jeu de renvois, de rappels, de réminiscences visuelles. Ce ne sont ni les mêmes gestes, ni les mêmes actions, ni les mêmes paroles qui sont répétés mais des mouvements, des traces qui s'inscrivent dans la composition des plans ou qui travaillent le fond des images. Prendre l'empreinte de la répétition dans ce qu'elle a de plus discret et de moins visible met au jour l'effet délicat et sensible qu'elle produit sur les images et sur la pensée. Ces reprises tissent un plan formel à la fois visible et invisible, très concret et pourtant furtif. Les personnages participent de la construction de ces fonds instables qui agencent le plan de consistance, mais Galoup qui en est tour à tour le motif, l'initiateur, le pivot ou le mobile est aussi celui qui en révèle les failles par son monologue intérieur et par les temps d'arrêt qu'il impose au déroulement des actions. Le tissu cinématographique que le film semble élaborer par des séries de lignes, de rythmes et de formes qui se conjuguent est ainsi régulièrement défait, contredit et contrarié comme dans ce plan sous-marin où, après une violente explosion 


\title{
hors champ, le cadre bleu se remplit de rouge jusqu'à en devenir vert, jusqu'au silence.
}

\author{
Université Aix-Marseille
}

\section{NOTES}

1. Concernant la méthodologie de la description des scènes, l'auteure s'est appuyée sur Svetlana Alpers (1990) et Jacques Aumont (1996).

2. Beau travail a d'ailleurs une large place dans l'ouvrage de Martine Beugnet (2012) où l'auteure analyse un nouveau rapport au corps et à la sensation dans le cinéma français des années 2000.

3. Pour une approche plus générale de la filmographie de Claire Denis, le lecteur pourra se référer à l'ouvrage de Rémi Fontanel, Le cinéma de Claire Denis (2008).

4. Certaines scènes sont préparées avec le danseur et chorégraphe Bernardo Montet.

5. Sur ce point voir l'étude de Laurent Guido, L'âge du rythme (2007).

6. "Entraînement, garde, lessives, repassage, sorties. La routine", dit l'adjudant Galoup dans le film.

7. Les scènes du début du film se déroulent au bord de l'eau, où le campement est installé. À plusieurs reprises les hommes se baignent ou s'entraînent dans l'eau. Puis un changement de camp les conduit vers des montagnes au-dessus de la côte, d'où la mer reste visible. Au large apparaissent trois petits îlots que désigne le monologue intérieur de Galoup: «Et trois volcans montaient la garde. Sentinelles.» Cet éloignement se poursuit lorsque Sentain, puni après être tombé dans le piège que lui tendait Galoup, se perd dans le désert et échoue, à bout de forces, au milieu d'une étendue salée. Le parcours du film se condense alors en un plan large. Il s'agit d'un plan d'ensemble vide en plongée sur la mer. Le panoramique glisse du bleu marine des grands fonds vers le bleu turquoise de la côte pour se terminer sur une plage de sel. Une minuscule silhouette entre dans le cadre, c'est Sentain, perdu, qui s'agenouille au bord de l'eau.

8. Ce résumé n'est pas faux, mais il ne ressemble pas au film. Beau travail pourrait être raconté différemment, selon une version tout aussi vraie. Pourtant, aucun résumé ne saurait dire sa réalité exacte, qui passe par un montage très sec et un enchaînement rapide de micro-événements.

9. De manière comparable, la relation de Galoup avec une jeune femme est traitée en deux plans. Au premier, assis à la terrasse d'un café, il achète un produit de beauté à un marchand ambulant. Au second, il glisse cette petite boîte dans la main de son amie allongée.

10. Lorsque, au cours d'une ronde de nuit, le commandant échange quelques mots avec lui, il lui demande s'il s'entend bien avec ses parents. Sentain répond qu'il n'a ni mère ni père et qu'il a été découvert bébé dans une cage d'escalier. Le commandant, surpris, s'exclame: "Trouvé? Oh! merde alors! Ah! Au moins ça a été une belle trouvaille.»

\section{RÉFÉRENCES BIBLIOGRAPHIQUES}

Alpers 1990 : Svetlana Alpers, L'art de dépeindre. La peinture hollandaise au XVII siècle, traduit de l'anglais par Jacques Chavy, Paris, Gallimard, 1990, 416 p. 
Aumont 1996 : Jacques Aumont, À quoi pensent les films, Paris, Séguier (coll. "Collection noire»), 1996, 287 p.

Beugnet 2012: Martine Beugnet, Cinema and Sensation: French Film and the Art of Transgression, Edinburgh, Edinburgh University Press, 2012, 192 p.

Deleuze 1968: Gilles Deleuze, Différence et répétition, Paris, Presses universitaires de France (coll. «Épiméthée»), 1968, 416 p.

Deleuze et Guattari 1980: Gilles Deleuze et Félix Guattari, Capitalisme et schizophrénie, tome 2, Mille plateaux, Paris, Minuit (coll. «Critique»), 1980, 645 p.

Fontanel 2008: Rémi Fontanel (dir.), Le cinéma de Claire Denis ou l'énigme des sens, Lyon, Aléas, 2008, 284 p.

Guerard 1928: Roland Guerard, "Le geste et le rythme», Cinéa - Ciné pour tous, $\mathrm{n}^{\circ} 112,1928$, p. 9-10.

Guido 2007: Laurent Guido, L'àge du rythme. Cinéma, musicalité et culture du corps dans les théories françaises des années 1910-1930, Lausanne, Payot, 2007, 544 p.

Kierkegaard 1984: Søren Kierkegaard, Crainte et tremblement. Lyrique-dialectique par Johannès de Silentio [1843], traduit du danois par P.-H. Tisseau, Paris, Aubier (coll. «Bibliothèque philosophique»), 1984, 217 p.

Melville 1987: Herman Melville, Billy Budd, marin [1924], Paris, Gallimard (coll. «L'imaginaire»), 1987, 182 p.

\section{ABSTRACT}

\section{Thwarted Regularities Caroline Renard}

Claire Denis's Beau travail is one of those films which invite us to view them from the point of view of repetition. A major aesthetic device of the twentieth century, repetition can also be a discrete form circulating from image to image. Sometimes it hides and disguises itself from one shot to the next in the same film. Using an analytical approach, the author employs the concept "plane of immanence" proposed by Gilles Deleuze and Félix Guattari in A Thousand Plateaus (1980) to engage in a figurative study of the kinds of repetition found in Beau travail. Deleuze and Guattari defined the "plane of immanence" as a formal space that is simultaneously a backdrop and a volume, a frame and a thread running through it. This space resists over time. In the cinema, the plane of immanence develops through the articulation of shots, the composition of the images and the movements that animate the space or the trajectories traversing it. It is a sometimes-receding visual bearing, in motion but locatable. The author's analysis of Beau travail demonstrates that its repetitions, far from simply constructing narrative or theatrical effects, contribute to the establishment of a perceptive base at once recurring and variable, a visual thread that is recognizable and unstable. She thus brings to light, in the figurative economy 
of this film inspired by literature and music, the establishment of this "plane of immanence" as the visible and invisible backdrop to film editing. 